\title{
PINK AND WHITE ESTHETIC SCORES OF POLYMER-INFILTRATED- CERAMIC-NETWORK AND LITHIUM DISILICATE IMPLANT HYBRID ABUTMENT CROWNS: RANDOMIZED CLINICAL TRIAL
}

\author{
Nourhan Elsaad Elrifaie*, Ahmed Naguib ${ }^{* *}$ and Reham El Basty ${ }^{* * *}$
}

\begin{abstract}
Objective: The aim of this study was to evaluate the effect of polymer-infiltrated-ceramicnetwork hybrid abutment crown on pink and white esthetics in comparison with lithium disilicate.

Materials and methods: Eighteen patients requiring single unit implant restorations in the maxillary esthetic zone were enrolled in the study. Each patient received a single screw-retained hybrid abutment crown of either lithium disilicate (IPS e.max CAD) or polymer-infilitrated ceramic network (VITA ENAMIC) and was followed up for 6 months. The pink and white esthetics were evaluated at 0,3 and 6 months timepoints using PES/WES index.
\end{abstract}

Results: Regarding the combined PES/WES and the PES, no statistically significant difference was found between IPS e.max CAD and VITA ENAMIC hybrid abutment crowns at any timepoint. However, regarding the white esthetic score, a statistically significant difference was found between the two groups, favouring IPS e.max CAD.

Conclusion: Within limitations of this clinical study, it could be concluded that IPS e.max CAD hybrid abutment crowns can yield satisfactory pink and white esthetic outcomes, when performed in well selected cases. VITA ENAMIC hybrid abutment crowns can yield clinically acceptable cumulative pink and white esthetic scores, however single elements of white esthetics, such as color, surface texture, translucency and characterization are significantly threatened by time.

KEYWORDS: dental implant, hybrid abutment crown, pink esthetic score, white esthetic score, PES/WES, lithium disilicate, VITA ENAMIC, hybrid ceramics

\section{INTRODUCTION}

Today, the "Branemark era" is over and the criteria for success in implant dentistry has changed from only obtaining successful osseointegration, to achieving complete biomechanical and esthetic integration of prosthetic and soft tissue frameworks. ${ }^{(1)}$ An ideal esthetic implant restoration is defined as a combination of a visually pleasing

\footnotetext{
* B.D.S., Faculty of Dentistry, Cairo University

** Professor of Fixed Prosthodontics, Faculty of Dentistry, Cairo University

*** Associate Professor of Fixed Prosthodontics, Faculty of Dentistry, Cairo University
} 
prosthesis and healthy harmoniously scalloped periimplant soft tissues. ${ }^{(2)}$

When it comes to the restoration of single implant-supported crowns, the maxillary esthetic zone remains a critical area. With patients' increasing esthetic demands, the evolution of highly esthetic implant solutions became a necessity.

Among the aspects of an implant superstructure that play a role in achieving the desired outcome are the esthetic materials used and the fabrication technique.

The use of CAD/CAM technology for implant reconstructions fabrication, has markedly facilitated and enhanced a process that is already complicated enough for both the patient and the clinical operator. $(3,4)$ Furthermore, simplified modern implant reconstructions, such as the hybrid abutment approach, which features a small titanium base to be bonded to a ceramic abutment, are only feasible with CAD/CAM. In addition, it allows the use of highly esthetic materials such as lithium disilicates and recently also the resilient hybrid ceramics, for example VITA ENAMIC. VITA ENAMIC is claimed to be ideal for implant restorations due to its ability to absorb masticatory forces, excellent compatibility with peri-implant tissues in addition to its good esthetics.

The seek for an ideal restoration material and design that balances the three major elements of a successful restoration, namely, mechanical, biologic and esthetic, will always create a reason for research. Therefore, the aim of this study was to evaluate the effect of polymer-infiltrated-ceramicnetwork hybrid abutment crown on pink and white esthetics in comparison with lithium disilicate.

The first null hypothesis was that polymerinfiltrated-ceramic-network hybrid abutment crown would give similar pink esthetic results when compared to the lithium disilicate hybrid abutment crown. The second null hypothesis was that polymer-infiltrated-ceramic-network hybrid abutment crown would give similar white esthetic results when compared to lithium disilicate.

\section{MATERIALS AND METHODS}

Eighteen patients requiring single unit implant restoration in the esthetic zone were selected from the outpatient clinic of Department of Fixed Prosthodontics, Faculty of Dentistry, Cairo University. Patients were selected following the inclusion criteria that required all patients to be:

1. Above 18 years old

2. Literate, capable of reading and signing the informed consent document

3. Healthy, free from or with controlled systemic disease

4. With acceptable oral health to support implant restorations

5. With good bone quality and quantity

6. Cooperative, accepting follow up and maintenance sessions

7. Having a single non-restorable tooth in the maxillary esthetic zone

Any patient not fitting one or more of the inclusion criteria had to be excluded. Pregnant women were also excluded due to the effect of pregnancy hormones on peri-implant soft tissues.

The eighteen participants were divided according to the restoration material into two groups: group (IPS e.max CAD) for lithium disilicate ceramic abutment crown and group (VITA ENAMIC) for polymer-infiltrated-ceramic-network abutment crown with 1:1 allocation ratio, nine participants each $(n=9)$.

Randomization was carried out using computerized sequence generation via www.random. org. The trial participants, outcome assessors and statistician were blinded throughout the research. 
Blinding of the researcher was not possible since she was responsible for all experimental procedures.

An informed patient consent was obtained from all patients who participated in the study under the supervision of the Ethics Committee of Faculty of Dentistry, Cairo University.

A thorough examination including cone beam computed tomography (CBCT) to the area of interest was performed. The tooth shade was determined using a visual shade guide (VITA Toothguide 3D-MASTER ${ }^{\circledR}$, VITA Zahnfabrik, Germany) according to the contra-lateral/adjacent tooth. Shade was also confirmed using a spectrophotometer (VITA Easyshade ${ }^{\circledR}$ V, VITA Zahnfabrik, Germany).

An immediate implant placement protocol was followed, where Neobiotech IS implant (Neobiotech, Korea) was used with different diameters and lengths according to the available bone volume in each case. The implant was left to osseointegrate for three months following the delayed loading protocol. Afterwards, a healing collar was placed for one week and then replaced by a screw-retained temporary restoration. Temporization took place until proper emergence profile, gingiva level and zenith were achieved. The soft tissue molding was done using the dynamic compression technique.

The digital solution was used for the hybrid abutment crown construction. Intraoral scanning was done using (CEREC Omnicam, Sirona Dental Systems). The soft tissue was scanned separately, then the titanium base was imaged by the aid of a scanbody attached to it. The triangular geometry of the scanbody guided the determination of the exact position, angulation and rotation of the inserted implant. The opposing quadrant and bite were also registered digitally.

Using CEREC 4.4 software, all dental and gingival scans were combined and used to create the entire virtual model of the restoration. The software proposed a restoration with proper contacts, occlusion, emergence profile, and tissue pressure based on the scanned gingival contours and emergence profile and the manufacturers' recommended restoration parameters of the different materials.

After final adjustments, the restoration was milled, according to the participant's group, from either a blue stage lithium disilicate (IPS e.max CAD A16 LT L, Ivoclar Vivadent) or a fully crystallized PICN (VITA ENAMIC IS-16 HT L, VITA Zahnfabrik) block of the correct shade using the milling machine (CEREC MC XL, Sirona Dental Systems). Because the restorations were designed as hybrid abutment crowns, they were milled in one piece to be screw-retained to the implant.

The restoration try-in was performed in the same visit of designing and milling. To facilitate the intraoral handling, the restorations were temporarily fixed into their positions on the titanium bases using light body addition silicone. For IPS e.max CAD restorations, the try-in was performed in the blue stage, while for VITA ENAMIC restorations, the try-in was performed in the fully crystallized state.

Modifications of the crowns were carried out using finishing diamond stones. For IPS e.max CAD restorations, the modified abutment crowns and digital intraoral photographs of the patients were sent to a laboratory technician for crystallization and staining. Combination firing technique was used, where crystallization and stain/glaze firing were performed in one step in a compatible ceramic furnace (Programat P310, Ivoclar Vivadent). The firing parameters for IPS e.max CAD LT were observed. For VITA ENAMIC restorations, the abutment crowns were individualized and stained according to the manufacturer's instructions, using a light-curing low-viscosity methacrylatebased filling composite (Te-Econom Flow, Ivoclar Vivadent) and light curing low-viscosity composite stains (Characterization Tints, Bisco). The surface of the VITA ENAMIC crown was treated using 
5\% hydrofluoric acid gel (IPS Ceramic Etching Gel, Ivoclar Vivadent) for 60 seconds, followed by bonding agent (Monobond Plus, Ivoclar Vivadent). The composite and stains were then added and completely light polymerized according to the manufacturer's instructions. Finally, the surface was sealed using a light-cured liquid polish (BisCover ${ }^{\mathrm{TM}}$ $\mathrm{LV}$, Bisco). The correct shade reproduction of the abutment crowns of both materials was confirmed using the visual shade guide and a spectrophotometer.

The abutment crowns were bonded to sterile titanium bases. The bonding surface of the titanium base was roughened using (A12O3) air abrasion and primed using a universal primer (Monobond Plus, Ivoclar Vivadent) for 60 seconds. The inner surfaces of the abutment crowns were etched using $5 \%$ hydrofluoric acid gel (IPS Ceramic Etching Gel, Ivoclar Vivadent) for $20 \mathrm{~s}$ and $60 \mathrm{~s}$, for lithium disilicate and VITA ENAMIC, respectively. Etched surfaces of both, lithium disilicate and VITA ENAMIC crowns were silanated using Monobond Plus. A self- curing luting composite resin (Multilink Hybrid Abutment, Ivoclar Vivadent) was used to bond the abutment crowns to the titanium bases, extraorally. After screwing of the hybrid abutment crowns to the implants, the screw channel was sealed using bonding agent and filling composite.

For evaluation of the study outcome Pink and White Esthetic Scores (PES/WES) according to Belser et al. $2009^{(5)}$, standardized intraoral photographs, showing the implant crown, the peri-implant soft tissues and at least one tooth on either side of the implant, were taken by the same photographer using two projections: one facial projection perpendicular to the facial implant crown and another incisal/ occlusal projection using a professional digital camera with $100-\mathrm{mm}$ macrolens and a dual-point flash. The occlusal view was used to assess the contour of the alveolar process. Photographs for the pink and white esthetic scores were taken the day of crown delivery, three months, and six months after. All photographs were exported to a computer with a high-resolution screen for viewing and evaluation.

The esthetic evaluation was performed by three independent observers, a periodontist, an orthodontist and a prosthodontist, who had not been involved in the treatment of the patients. The assessors were also blinded regarding the presence of three time points. Their evaluation was conducted according to the PES/WES index. The PES is comprised of the following variables: mesial papilla, distal papilla, curvature of the facial mucosa, level of the facial mucosa, root convexity, and soft tissue color and texture at the facial aspect of the implant site. The WES includes five variables: tooth form, tooth volume, tooth color (including assessment of hue and value), surface texture and translucency and characterization. A score of 2,1 , or 0 was assigned to each parameter. The parameters were assessed by direct comparison with the natural, reference tooth, estimating the degree of match or eventual mismatch. The threshold of clinical acceptability was $6 / 10$ for each component PES or WES, thus a $\mathrm{PES} / \mathrm{WES} \geq 12$ was considered as the limit for an acceptable esthetic outcome of implant treatment, and a score of 9 or more for each component or a PES/WES $\geq 18$ was considered almost perfect. ${ }^{(5,6,7)}$

\section{RESULTS}

All patients attended all follow up sessions in the control group (Group IPS e.max CAD; $n=9$ ) and in the intervention group (Group VITA ENAMIC; $n=9$ ). Study data were collected at the time of hybrid abutment crown delivery (baseline), (3 months) and (6 months) after hybrid abutment crown delivery. For statistical analysis, the mean and standard deviation values were calculated for each group in each test. Normality distribution was tested using Kolmogorov-Smirnov and Shapiro-Wilk tests, which revealed that data were not normally distributed (non-parametric). Friedman test was 
used to compare between more than two groups in related samples. Wilcoxon test was used to compare between two groups in related samples, while Mann Whitney test was used to compare between two groups in non-related samples. The significance level was set at $\mathrm{P} \leq 0.05$. Statistical analysis was performed with IBM ${ }^{\circledR}$ SPSS ${ }^{\circledR}$ Statistics Version 20 for Windows. Regarding the pink esthetic score, no statistically significant difference was found between (IPS e.max CAD) and (VITA ENAMIC) at baseline, after 3 months or after 6 months, where $(p=0.953,0.224$ and 0.461$)$, respectively. Similarly, no statistically significant difference was found, regarding the white esthetic score, between (IPS e.max CAD) and (VITA ENAMIC) at basline or after 3 months where ( $p=0.905$ and 0.367$)$, respectively. However, after 6 months, a statistically significant difference was found between the two groups where $(p=0.012$ ), favoring IPS e.max CAD. For the total pink and white esthetic score, no statistically significant difference was found between (IPS e.max CAD) and (VITA ENAMIC) at baseline, after 3 months or after 6 months, where $(p=0.839$, 0.792 and 0.268 ), respectively.

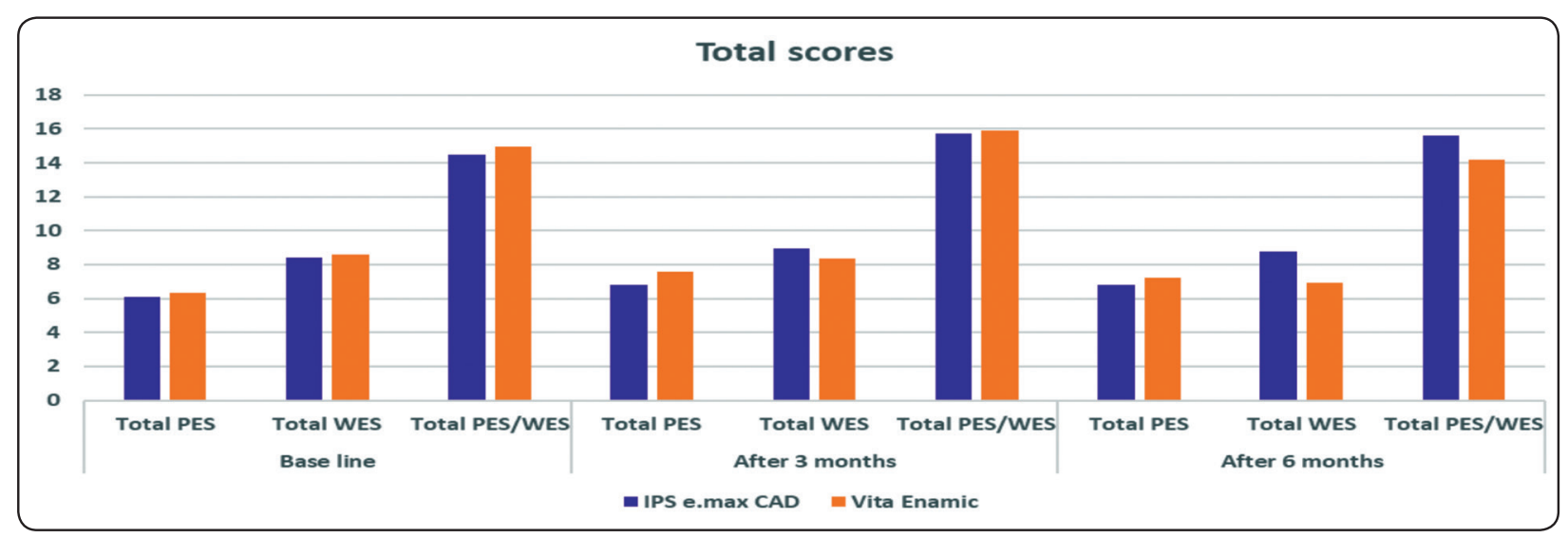

Fig. (1): Bar chart representing effect of materials in different time periods.

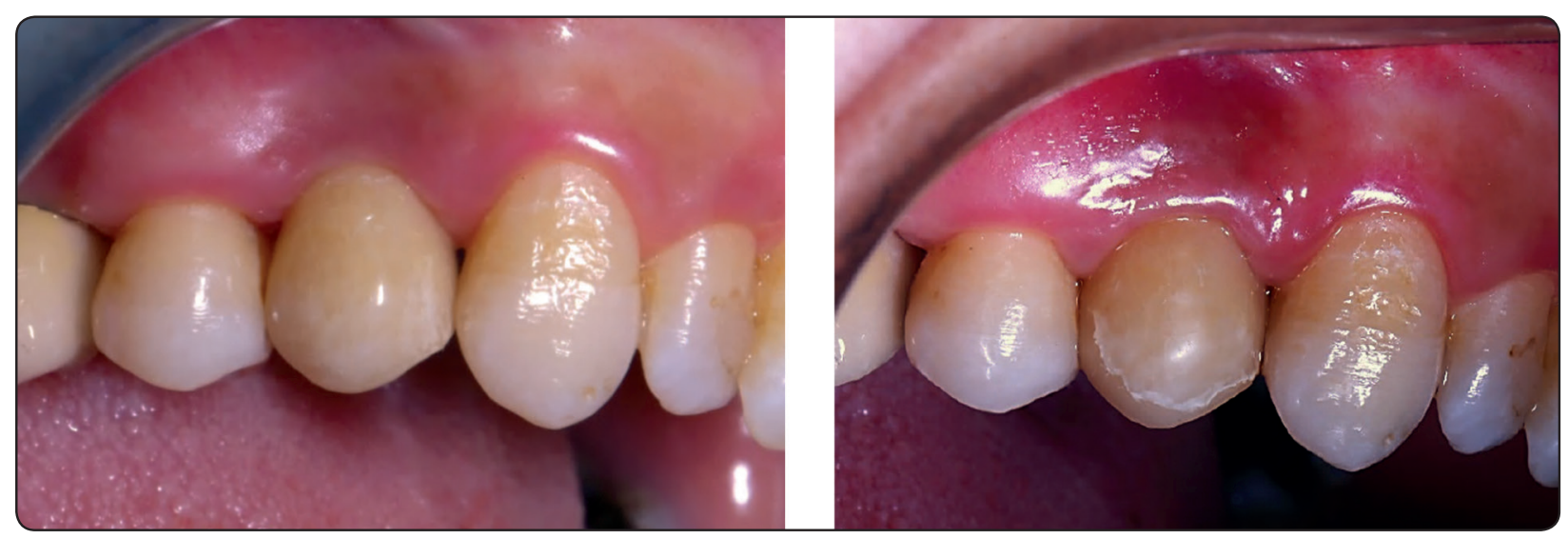

Fig. (2): A VITA ENAMIC abutment crown (upper right 4 position) at 0 (left) and 6 months (right) follow up. 


\section{DISCUSSION}

With respect to pink tissue evaluation, no statistically significant difference was found between (IPS e.max CAD) and (VITA ENAMIC) at any timepoint. It was expected that VITA ENAMIC would have a more favorable effect on the soft tissues, based on a previous finite element analysis by Bijjargi et al., in 2013, ${ }^{(8)}$ which measured the stress dissipation in bone through various crown materials of dental implant restorations and concluded that an occlusal material with a low modulus of elasticity, dampens the occlusal impact forces and therefore decreases the effect on bone-implant interface, However, the very short follow up period in the current study, was not enough to show and correlate the effect of bone resorption on the soft tissue level. Nevertheless, an abrupt increase in the pink esthetic scores was found in the VITA ENAMIC group during the first three months, which could be related to the biocompatibility of the material with the periimplant tissues.

A recent study by Astasov-Frauenhoffer et al., in 2018, ${ }^{(9)}$ reported that VITA ENAMIC showed a statistically significantly lower biofilm formation than zirconia. This was explained by its organic content. However, a contradicting decrease of scores was found from (3 months) till (6 months) which has been homogenous with a detectable partial loss of the applied stain layer. This detachment of stain layer has probably caused an increased surface roughness. It was reported that rougher surfaces with a Ra value above $0.2 \mu \mathrm{m}$ are shown to promote bacterial adhesion. This could have led to increased plaque accumulation, gingival inflammation of the very sensitive peri-implant tissues and thus papillary regression. The partial loss of stain layer in the (VITA ENAMIC) group has also negatively influenced the surface texture and thus the white esthetic score, where a significant difference was found between the two groups only after 6 months, favouring (IPS e.max CAD).
In agreement with this study, Mühlemann et al., in 2018, ${ }^{(10)}$ showed using SEM analysis that the staining layer on VITA ENAMIC formed an inhomogenous surface and that it was partially removed over time due to the action of tooth brushing. It was thus concluded that the applied staining protocol significantly increased surface roughness of VITA ENAMIC.

Another parameter that played an important role in the white esthetic score of the current study is tooth color which also includes hue and value. The initial shade reproduction of VITA ENAMIC restorations was more difficult due to two main reasons. First, the available VITA ENAMIC mesoblock shades are limited when compared to IPS e.max CAD mesoblocks. Only five shades (1M1, 1M2, 2M2, $3 \mathrm{M} 2,4 \mathrm{M} 2$ ) are available for VITA ENAMIC (11) while for IPS e.max CAD ten shades (BL2, A1, A2, $\mathrm{A} 3, \mathrm{~A} 3.5, \mathrm{~B} 1, \mathrm{~B} 2, \mathrm{C} 1, \mathrm{C} 2, \mathrm{D} 2)$ are available. ${ }^{(12)}$ The second problem was the technique-sensitivity of the shade individualization protocol of VITA ENAMIC restorations, where chemical conditioning of the surface sometimes left white patches on the surface that were not concealable by the staining materials. It was obvious that ENAMIC restorations got stained during the follow up period as opposed to IPS e.max CAD. ENAMIC comprises of a porous ceramic network that is reinforced, via capillary action, with a polymer matrix. The ceramic network may be exposed if the polymer matrix degrades, leading to rougher surfaces which are more prone to stain uptake. ${ }^{(13)}$

In accordance with our results, Sarikaya et al., in 2018, ${ }^{(14)}$ concluded that the color stainability of VITA ENAMIC by coffee and tea was beyond the clinically acceptable threshold, which was related to its TEGDMA content. Discoloration of VITA ENAMIC was also reported with other colored nutrients, such as cress and curry. ${ }^{(15)}$ 
On the other hand, IPS e.max CAD abutment crowns were relatively resistant to discoloration during the follow up period. In accordance with our findings, Stawarczyk et al., in 2016, (15) reported that IPS e.max CAD showed a statistically significantly lower discoloration rate compared to VITA ENAMIC, regardless of the storage medium. In addition, Alp et al. in 2018, ${ }^{(16)}$ concluded that when glazed lithium disilicate is subjected to coffee thermocycling, the color change was not perceptible.

Another factor influencing the white esthetic outcome is the inherent translucency parameters of the materials. IPS e.max CAD has a statistically significantly higher translucency parameter than VITA ENAMIC with a reported mean of 26.0 \pm 0.6 and $16.0 \pm 0.6$, respectively. ${ }^{(17,18)}$ Although, as recommended by the manufacturers of both materials, a high translucency (HT) block was used for VITA ENAMIC and a low translucency (LT) block was used for IPS e.max CAD, the visible translucency of IPS e.max CAD was still higher.

In accordance with our scores, Stawarczyk et al., in 2016, ${ }^{(15)}$ reported that VITA ENAMIC showed a significantly lower translucency than lithium disilicate ceramic. The investigator failed to reject the first null hypothesis, since no statistically significant difference was found between the two groups at any timepoint, concerning the pink esthetic score. While for the white esthetic score, a statistically significant difference was found between the two groups, favouring IPS e.max CAD, and therefore, the second null hypothesis was rejected.

\section{The limitations of this study:}

1. The relatively short follow-up period of 6 months forms limitation of the trial.

2. The PES/WES esthetic index created some limitation in the current study. The three points scoring system is very limited and highly influenced by subjectivity.

\section{CONCLUSION}

Within limitations of this clinical study, the following conclusions could be drawn as follows:

1. IPS e.max CAD hybrid abutment crowns can yield satisfactory esthetic outcomes, when performed in well selected cases.

2. Based on a combined judgement of pink and white esthetic scores, VITA ENAMIC hybrid abutment crowns can yield clinically acceptable esthetic outcomes. However, single elements of white esthetics, such as color, surface texture, translucency and characterization are significantly threatened by time.

\section{CLINICAL RECOMMENDATIONS}

1. The staining protocol of VITA ENAMIC negatively influenced the restoration esthetics over time. It is recommended to develop improvement of the characterization technique of VITA ENAMIC restorations.

2. Further randomized clinical trials are recommended to evaluate the pink and white esthetics of IPS e.max CAD and VITA ENAMIC hybrid abutment crowns for more prolonged follow-up periods.

3. A more comprehensive esthetic index such as Implant Restoration Esthetic Index (IREI) is recommended for the evaluation of pink and white esthetics.

\section{CONFLICT OF INTEREST}

The authors report no conflict of interest related to this study.

\section{REFERENCES}

1. Buser, D., Martin, W., \& Belser, U. C. (2004). Optimizing esthetics for implant restorations in the anterior maxilla: anatomic and surgical considerations. International Journal of Oral \& Maxillofacial Implants, 19(7).

2. Wittneben, J. G., Buser, D., Belser, U. C., \& Brägger, U. (2013). Peri-implant soft tissue conditioning with 
provisional restorations in the esthetic zone: the dynamic compression technique. Int $\mathrm{J}$ Periodontics Restorative Dent, 33(4), 447-455.

3. Abduo, J., \& Lyons, K., (2013). Rationale for the Use of CAD/CAM Technology in Implant Prosthodontics. International Journal of Dentistry, 2013, 8 .

4. Mühlemann, S., Kraus, R. D., Hämmerle, C. H., \& Thoma, D. S. (2018). Is the use of digital technologies for the fabrication of implant-supported reconstructions more efficient and/or more effective than conventional techniques: A systematic review. Clinical oral implants research, 29, 184-195.

5. Belser, U. C., Grütter, L., Vailati, F., Bornstein, M. M., Weber, H. P., \& Buser, D. (2009). Outcome evaluation of early placed maxillary anterior single-tooth implants using objective esthetic criteria: a cross-sectional, retrospective study in 45 patients with a 2-to 4-year follow-up using pink and white esthetic scores. Journal of periodontology, 80(1), 140-151.

6. Arora, H., \& Ivanovski, S. (2018). Evaluation of the influence of implant placement timing on the esthetic outcomes of single tooth implant treatment in the anterior maxilla: A retrospective study. Journal of Esthetic and Restorative Dentistry.

7. Mangano, F. G., Mastrangelo, P., Luongo, F., Blay, A., Tunchel, S., \& Mangano, C. (2017). Aesthetic outcome of immediately restored single implants placed in extraction sockets and healed sites of the anterior maxilla: a retrospective study on 103 patients with 3 years of followup. Clinical Oral Implants Research, 28(3), 272-282.

8. Bijjargi, S., \& Chowdhary, R. (2013). Stress dissipation in the bone through various crown materials of dental implant restoration: a 2-D finite element analysis. Journal of investigative and clinical dentistry, 4(3), 172-177.
9. Astasov-Frauenhoffer, M., Glauser, S., Fischer, J., Schmidli, F., Waltimo, T., \& Rohr, N. (2018). Biofilm formation on restorative materials and resin composite cements. Dental Materials, 34(11), 1702-1709.

10. Mühlemann, S., Bernini, J. M., Sener, B., Hämmerle, C.H., \& Özcan, M. (2018). Effect of Aging on Stained Monolithic Resin-Ceramic CAD/CAM Materials: Quantitative and Qualitative Analysis of Surface Roughness. Journal of Prosthodontics.

11. www.vita-zahnfabrik.com

12. www.ivoclarvivadent.us

13. Quek, S. H. Q., Yap, A. U. J., Rosa, V., Tan, K. B. C., \& Teoh, K. H. (2018). Effect of staining beverages on color and translucency of CAD/CAM composites. Journal of Esthetic and Restorative Dentistry, 30(2), E9-E17.

14. Sarıkaya, I., Yerliyurt, K., \& Hayran, Y. (2018). Effect of surface finishing on the colour stability and translucency of dental ceramics. BMC oral health, 18(1), 40.

15. Stawarczyk, B., Liebermann, A., Eichberger, M., \& Güth, J. F. (2016). Evaluation of mechanical and optical behavior of current esthetic dental restorative $\mathrm{CAD} /$ CAM composites. Journal of the mechanical behavior of biomedical materials, 55, 1-11.

16. Alp, G., Subasi, M. G., Johnston, W. M., \& Yilmaz, B. (2018). Effect of surface treatments and coffee thermocycling on the color and translucency of CAD-CAM monolithic glass-ceramic. The Journal of prosthetic dentistry.

17. Gunal, B., \& Ulusoy, M. M. (2018). Optical properties of contemporary monolithic CAD-CAM restorative materials at different thicknesses. Journal of Esthetic and Restorative Dentistry.

18. Sen, N., \& Us, Y. O. (2018). Mechanical and optical properties of monolithic CAD-CAM restorative materials. The Journal of prosthetic dentistry, 119(4), 593-59. 